\title{
Moving from VR into AR using Bio-Cybernetic Loops and Physiological Sensory Devices for Intervention on Anxiety Disorders
}

\author{
Preyesse Arquissandás . David Ribeiro Lamas . Jorge Oliveira
}

Received: date / Accepted: date

\begin{abstract}
Anxiety disorders comprise different clinical conditions that affect individuals in their personal, professional and social domains. The development of new intervention approaches for the treatment of anxiety disorders is crucial. As a step forward into promoting the well-being through adaptive physiological responses, we developed an Augmented Reality (AR) based system using bio-cybernetic loops to create an adaptive system for exposure therapy in anxiety disorders. The system was built using open source software (e.g., NyARToolkit, and Unity 3D). AR technology uses computer-generated information to enrich the real world. It can be used with less intrusive devices to collect physiological data (e.g., Bitalino) describing human behavior in a cycle. In this context our research project aims to study behavior during exposure to biologically relevant stimuli such as snakes. Phobia is described as an irrational fear to an object/stimulus. This fear triggers several physiological responses from sensors as increased heart rate (ECG) and skin conductance (EDA), which are responses from the autonomous nervous system. This approach can be used in several sessions, where the system through machine learning algorithms adapts the tresholds to the individual profile of each participant from historical data. Our study has been carried out in two stages: (1) The participants in a total of 35 students (30 males and 5 females with ages ranging from 19 to 29 years) were invited to fill a snake questionnaire (SNAQ). (2) A sub-sample was enrolled in an exposure session in AR using a virtual snake while collecting psychophysiological responses from sensors data. The results have shown increased physiological responses in two AR exposure sessions using snakes as stimuli. Therefore we conclude that the system was efficient to detect changes in physiological responses during the exposure sessions.
\end{abstract}

Keywords Augmented Reality . Adaptive Systems . NyARToolkit . Unity 3D . Bitalino

\section{Introduction}

Nowadays, Virtual Reality (VR) technology is used in variety of fields (Parsons \& Rizzo, 2008), products and areas such as Education, Military, Medical, and Gaming (Gamito et al., 2011; Van Rooij et al., 2016), where each of the participants is fully immersed in a virtual environment. On the other hand, Augmented Reality (AR) (Baus $\&$ Bouchard, 2014) move each of those participants into a next level of interaction, where real and virtual worlds are combined. Such combination allows them to interact and manipulate the virtual objects using external stimulus.

The solution package specifically developed for this study is a suite of integrated applications that a therapist can use to collect, store, manage and interpret data from sensory devices during the therapeutic sessions

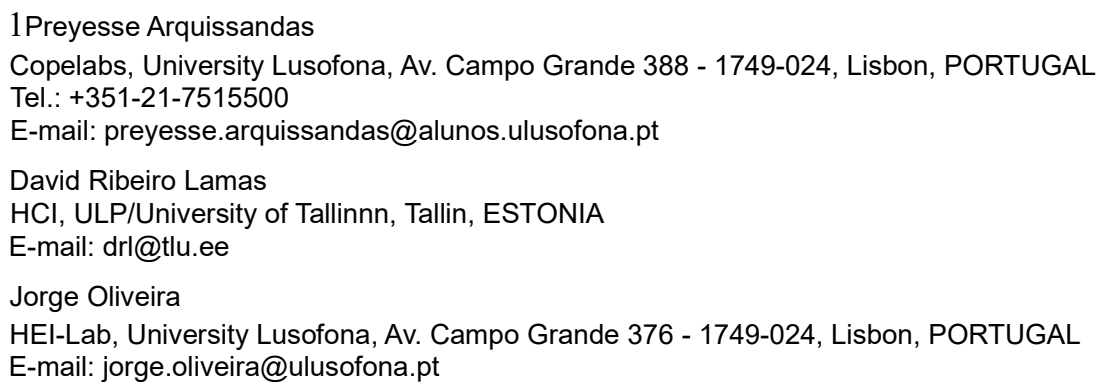




\section{Noname manuscript No. \\ (will be inserted by the editor)}

(Arquissandás, Lamas, and Oliveira, 2019). One of such device is the Bitalino, which reads electrical signals from sensors, connected to each of the participants body and allows to transform those signals into meaningful data through classification procedures of the input signal. To continuously read those signals and extract features, we used a systematic and recurring methods based on bio-cybernetic loop process (Muñoz et al., 2017). The extracted features with meaningful data are then presented on screen to each participant, using an Augmented Reality (AR) software (i.e., NyARToolkit) and a 3D modeling software package (i.e., Unity 3D). The programming language used for development was $\mathrm{C \#}$ for mono develop in Unity 3D. The compilation was done using .Net framework 3.5. This solution was prepared to run on a variety of platforms such as Windows and Android, with the use of an internal database for historical data storage such as SQLite 3.8 and Flat file - CSV.

The main requirements we had when initially identified hardware and software for this solution, were the following: (1) The need to be non-intrusiveness; (2) The need to offer a low cost solution; (3) The need to offer a reusable hardware platform; (4) The need to use available free or open source software with focus on community help; (5) The simplicity of integration and ease of development process; (6) System performance; and (7) Interoperability.

This paper aims to describe a technological solution for the exposure therapy (Suso-Ribera et al., 2019) in a anxiety disorders (Botella, 2004). In this solution, the key players are the therapists and the participants (Riva, Molinari, and Vincelli, 2002). In this approach, besides the clinical assessment and therapy, the therapists conduct the therapeutic sessions plugging each of the participants to the system and controlling the evolution among multiple sessions in terms of psychophysiological responses, using historical data stored on each of the session. The positive results are given by the decreases in psychophysiological activation to the stimulus and the ability to interact with such object in the augmented reality. For the purpose of this study, we assessed a group of participants without history of anxiety disorders. The selected group of 35 participants filled the Snake Questionnaire - SNQ (Klorman et al., 1974) and signed the informed consent regarding data privacy. The SNQ had some direct questions with ranging score of 0 or 1 where 0 means negative|false and 1 means positive|true. There were also some questions evaluated in a reverse order for validity assessment. The participants were first selected from the SNQ self-report results and involved in the second stage of the study, which consisted off plugging the sensor device for psychophysiological data capture, while the participants were subject to a brief exposure session of 20 minutes. One of the sensors read electrocardiogram (ECG) signals (Gacek \& Pedrycz, 2011) which measured the heart rate activity, while the other sensor read electrodermal activity (EDA) (Muñoz, Gouveia, Cameirão, and Badia, 2017) which measured the skin conductance. The combination of both sensors actuators, results in an overall signal reading from activation status of participants stimulus related to electrical activity when the participants were exposed to biologically relevant stimulus as a snake that triggers fight-orflight responses. All the procedures and exposure sessions are non-intrusive. The solution system is adaptive, scalable, as well as portable. The expected results are toward a decrease of electrical activity over consecutive sessions and an increased interactivity with fear cue, indicating a decrease in fear response to snake stimuli.

\subsection{Problem statement}

A Virtual Reality (VR) system drive each of the participants into a very limited and confined environment, where the experience is engaged in an synthetic virtual world on which the participant is immersed (Powers \& Emmelkamp, 2008). On such environment, the participants lose perception of the surrounding real world. To overcome this problem and take them into a journey of interaction sensations while experiencing the real world, the Augmented Reality (AR) (Wiederhold, 2012) was the choice. On such environment the participants can interact with the fear cue that otherwise would not be possible to manipulate (i.e., representation of a virtual snake in opposite to real one, due to the danger it represents, as well as the possibility of addressing even greater arousal to the participant) (Emmelkamp el al, 2002). This approach (Wrzesien, Burkhardt, Alcañiz, and Botella, 2011) allows the therapists to assist in real time all the reactions the participants are experiencing during the therapeutic sessions and validate if they are responding positively to the fear cue by using the method of crossmodal exposure when visually facing and touching the fear cue object. To accomplish such task, it is necessary to develop a predictive system for the treatment of specific phobias (Wolitzky, Horowitz, Powers, and Telch, 2008). Phobias are classified in the umbrella of anxiety disorders, being a mental disorder, according to the DSM 5 (APA, 2013). The treatment of such mental disorders involves exposure therapy (Duenser, 2014), which is usually conducted on a gradual and systematic basis. The content of the therapeutic sessions changes throughout treatment in order to meet the specific needs of the patient at each treatment stage. To meet these conditions, the therapist needs to evaluate the participant's responses in the previous sessions to define the content of the subsequent sessions. 
Noname manuscript No.

(will be inserted by the editor)

\subsection{Objectives}

The main objective of this paper is to provide to a suite of internal tools that assist therapists and participants in the treatment process for phobias, by avoiding time consumption spent by the therapists in patient assessment, and also to make this assessment less prone to the human error when conducting phobia treatment. In order to achieve this goal, first our intent was to build an adaptive, user-friendly and non-intrusive solution to study psychophysiological responses to fear relevant stimuli in immersive AR exposure. This solution must apply stimulus to the participants using a virtual object as well as a real object for crossmodal exposure sensation. Second, the solution to build should provide an interface capable of reading psychophysiological response data from peripheral devices (i.e., sensors) and register historical data on physical support (i.e., database, flat file). Finally, the solution must be able to generate reports based on session progression, export data to the external support devices for later review and analysis, as well as maintain a detailed log of all the tasks during the therapeutic sessions.

\section{Background}

\subsection{Traditional Treatment}

The treatment of certain phobias, may rely on different methods. One of such method is the Cognitive Behavioral Therapy (CBT) which is a combination of Cognitive Therapy and Behavior Therapy. In this type of therapy, the therapists identify the maladaptive cognitions and replace them therapeutically with adaptive cognition (Emmelkamp et al., 1992). Since the human behaviors and feeling may be a result from mental activities and external events or the interaction between both, one of the benefits of such condition is the ability to change the mental state in order to reflect changes in thinking and behaviors facing same situation. Therefore, each of the participants can be put on a imagery anxiety-challenging or demanding situation in order to identify unrealistic thoughts, trigger those thoughts, and replace these maladaptive thoughts by adaptive cognitions in their place.

\subsection{Exposure Treatment}

The exposure therapy is used on participants suffering from phobia. On this type of therapy, the exposure to anxiety challenging or demanding situations is carried out gradually in order to allow memory replacement from old anxiety experiences. The exposure therapy can be performed in vivo which means that it is done in real life with real life situations or in vitro, which means that it is done by imagining the anxiety challenging or demanding situation. According to some research (Botella et al., 2016) the in vivo therapy is more effective than in vitro when treating phobias. Other research points to the negative side of the use of in vivo therapy, due to the fact that the participants find it more challenging or demanding when dealing with the real life situation, therefore impacting on treatment adherence (Frans, 2004).

\subsection{AR System for Treating Anxiety Disorders through psychophysiological assessments}

There are a variety of studies using AR systems with success for the specific phobias treatments, such as phobias for the small animals (cockroaches, spiders and snakes). Some of these studies are focused on evaluating the AR system efficacy (Juan and Calatrava, 2011; Juan and Joele, 2011), while others are focused on the environment side (Wrzesien et al., 2011a) and the sense of presence (Juan and Prez, 2010). Overall studies seem to point to $\mathrm{AR}$, being a promising and useful tool for interventions in the treatment of specific phobias where each of the participants find themselves in a safe environment under therapist's supervision, while they are subjected to experiments with changing situations, such as the exposure to fear or phobic stimuli. In our study, the electrocardiogram (ECG) and electrodermal activity (EDA) signals of each of the participants are measured during the AR exposure sessions. The HRV - heart rate variability signal measures the heart's electrical activity over a period of time, which reflect arousal associated with emotional responses. These changes in HRV are controlled both by sympathetic and parasympathetic divisions of the autonomic nervous system. Combining different psychophysiological techniques, it is possible to identify human emotions and mental stress (Agrafioti et al., 2012; Rattanyu and Mizukawa, 2011; Ohkura et al., 2011; Harada et al., 2014). The Galvanic Skin Response (GSR) via EDA measures the changes in the electrical conductivity properties of the skin, caused by the sweat glands (which are controlled by the sympathetic division of the autonomic nervous system), when they are subjected to higher (fear and excited) or lower (sad and relaxed) stimuli. Due to these properties, the EDA (or electrodermal activity) is used to measure physiological arousal levels and mental stress (Kim et al., 2004; Nakasone et al., 2004). 


\section{Noname manuscript No.}

(will be inserted by the editor)

\subsection{Anxiety-AR Application Literature}

The current solutions available for the treatment of anxiety disorders are based on Virtual Reality (VR), and most without the aggregated solutions for sensor data collection. In Virtual Reality (VR), each of the participants are normally immersed into a reality from which he/she is not able to perceive the real world around and interact with a physical fear cue. In order to improve their experience with real world, the Augmented Reality (AR) solution was the choice, because it allows direct visualization of the world and, at the same time, to interact with the fear cues by its projection into current field of view (FOV). Moreover, the therapist can have access to the same FOV as the participant and monitor his progress throughout treatment sessions. The therapist is also able to monitor not only the body language, but also the participant interaction and behavioral and non-behavioral responses to stimulus provided during the sessions. Since the sessions configurations changes in an adaptive manner, there is no need to remember instructions, keeping the system free of error. It would be very hard to track participants performance and also to generate reports without the properties described for this system. The Anxiety-AR is programmed to perform an adaptive session with participants suffering from the specific anxiety disorder, such as snake phobia. The procedures followed to start these sessions are the following: 1) Start application and choose the intervention session for the particular participant; 2) register each of the participant into the system; 3) inform the participant, what the session is about and what is expected to do; 4) program each of the session duration time; 5) follow the progress of each participant during the therapeutic session; and 6) analyze the final report of overall session success.

\section{Methods and materials}

We have stepped into multiple phases to address the correct approach toward the AR solution preparation, such as: (1) Requirements Phase - The early version of the documentation was produced, taking in consideration the architecture to be validated in the System Design Phase. (2) System Design Phase - This phase represents the design of a prototype and all the aspects that needed in terms of hardware and software requirements to accomplish the next phase. The initial documentation was also updated in order to describe the procedures. (3) Development Phase - The first working prototype was assembled and a proof-of-concept (POC) carried out with success. For this test, we have submitted each of the participants to the AR solution by projecting snake images and measuring the feedback responses from sensors data collected using external sensory device for ECG//HRV and EDA data capture. (4) Transition / Training Phase - The Transition/Training Phase, intended to expand the initial test group, for more than one participant in order to achieve more reliable results. This study uses different data collection methods such as: the survey data collected from questionnaire, and the psychophysiological data collected from sensory device. The document was updated, with survey data collected from questionnaire and their findings. The Architectural Goals and Constraints of AR solution were related to: The use of psychophysiological responses from the participants during the sessions through peripheral sensors (i.e., ECG, EDA), but also related to collecting the participatory input through a visual analog scale (i.e., tactile feedback input system, avoiding large hands movements and interruptions during ongoing session) as an indicator of the subjective experience of emotions during the session (i.e., figures depicting different states adapted from the Self-Assessment Manikin (SAM) (Bradley and Lang, 1994)). Moreover, the concerns with the General Data Protection Regulation (GDPR) security issues of the participants data access were also continuously assessed during this implementation, making it highly restricted and anonymized in such way that there is no direct linkage between the participants and his/her psychophysiological data. This link was done using a numeric code that was only available to the researcher responsible for collecting the data. The build process followed some guiding principles in order to satisfy standards and measurements. One of those principles was the design aspect in order to validate and align with global architecture. Other principles were the scalability in both direction (i.e., horizontally by adding more sensors and vertically by adding more hardware resources, as memory, power lifetime, extra communication peripherals). In terms of software, it can be extended in order to incorporate other AR platforms or frameworks. The AR solution, can be flexible enough to accommodate new requirements without affecting the existing ones. In order to allow for the easy integration of the new features within the application, the AR solution was built on a modular architecture. The AR solution complies with the standard in the application developments, as well as with the design and the architecture. As an example of the software standards, it includes .Net, C\#, Unity 3D. As an example of the hardware standards, it includes Bitalino, Atmel Chip. The design and development of the AR solution, uses Unity 3D/Mono Develop. As object oriented software, it allows the use of elements of the design patterns. These elements can be reusable, allowing easy adaptation in the particular context. Since these elements are commonly used by the developers, most issues, as well as pros and cons of the implementation were well known beforehand. 
Noname manuscript No.

(will be inserted by the editor)

\subsection{Participants}

In order to analyze the usage of this application as an AR exposure technique in the context of phobia treatment, we have divided the tasks in two phases: (1) The participants in a total of 35 university students (30 males and 5 females with ages ranging from 19 to 29 years, $M=23$ years, $S D=10,22$ ) were invited to fill a snake questionnaire (SNAQ) as in Table 1 below. (2) Enroll each of those participants later on, in a Exposure intervention session to collect psychophysiological responses of ECG and EDA, in a presence of the fear cues using AR and sensory technology. According to overall participants electrical activity levels and arousal feedbacks, some messages and audio feedback are sent to the participant in order to encourage him to a relaxation state (i.e, "Stay calm", "Take deep breath", "Would you like to stop the session?").

\section{Table 1: Snake Questionnaire (SNAQ) Sample - (Klorman et al., 1974).}

\subsection{SNQ Questionnaire}

The questionnaire results are shown in Table 2 below. The participants were recruited from a university campus, and they have participated on a voluntary basis after filling the consent agreement. We have found a variety of sociodemographic coverage:

\section{Table 2: The Questionnaire overall sociodemographic coverage.}

The Snake Questionnaire consists of 30 self-reported questions which measures the level of the fear and phobia of snakes. It uses (true; false) dichotomous responses format, with "True" responses summarizing a score ranging from 0 to 30. It also has 9 reverse-scored questions (numbers 6, 12, 14, 16, 17, 20, 25, 27 and 28) which was not accounted, since those questions are harder to answer correctly, and do not appear to measure the same phenomenon. The Fig. 1 and Fig. 2 shows the sociodemographic distribution according to question number.

\section{Figure 1: Summary responses of SNQ by Origin/Gender.}

\section{Figure 2: Sociodemographic data.}

The Exposure intervention session to collect psychophysiological responses of ECG and EDA in a presence of the fear cues using AR and sensory technology, was performed with each of the participants (Table 3). The participant was subject to a brief interview to introduce to the session topics and fill the SNQ. The next step was to plug the sensory device into specific regions of the body for collecting the ECG and EDA signals in four distinct 5 minutes assessment moments. The first assessment moment was defined as the first baseline, to measure signals during a relaxation state. The second moment was the AR session to measure activation during exposure to a virtual snake during visual interaction. The third moment was the second baseline to measure relaxation following the activation phase of AR exposure. Finally, the fourth moment was the crossmodal session with a rubber snake to measure activation to tactile and visual interactions. The analysis of the data obtained from ECG and EDA indicates that electrical signals values changes according to the task condition (baseline, activation tasks) which suggest that this system is effective in capturing psychophysiological changes. The Fig. 3 shows data sample from these assessment moments.

\section{Table 3: The Snake Questionnaire (SNQ) results of the participants.}

Figure 3: Psychophysiological data sample from assessments moments.

The result from each of four assessment moments are compiled in order to show the overall progress in a 20 minutes session. The Fig. 4 shows that the participant in session (1) and session (3) had several activations due to stress level variations as in Fig. 5. In session (2) the same participant has achieved the target hit (red smile) which mean that the snake killed the mouse as in the Fig. 6 . The overall purpose of the activation sessions was to avoid the snake of killing the mouse by lowering activation to baseline state. Finally in the session (4) there was an activation due to fear cue while handling it physically as in the Fig. 7. 
Noname manuscript No.

(will be inserted by the editor)

Figure 5: Baseline sessions (1,3).

Figure 6: AR session (2).

Figure 7: SNAKE session (4).

\subsection{AR Exposure}

The AR exposure session was carried out in a one-session intervention related with fear of snakes for each of the participant. During the session, the ECG and EDA sensors were attached to the participant and the AR application system was initialized. After the initialization, the system presents the fear cue and starts collecting the electrical signals related to ECG and EDA. The observation was done in three steps: (1) In the first step the participant is instructed to relax and watch some neutral images such as (i.e., geometric figures, programming languages, animals, and math formulas) while the baseline signals are collected. (2) In the second step the participant is instructed to lower down the anxiety level in order to avoid the mouse being captured by the snake, by controlling the breathing and try to relax, while watching the AR snake interaction with the mouse. (3) In the third step the participant is instructed to play with a rubber snake while the signals are collected.

\subsection{Architecture Diagram}

The below diagram in Fig. 8 illustrates the AR solution architecture and the communication between different components.

\section{Figure 8: Architecture Diagram.}

The main component of the AR solution, use camera orientation to collect images from webcam. The collected images are subjected to a rendering process by which the image is segmented, and features are extracted. Finally, the image is augmented by a marker detection. The Unity 3D is responsible for providing the application component and visual presentation. This component, also reads data from external devices and manage the use and storage of such data by AR system. The visual presentation part allows the interaction between the participant and the AR system. The interaction is initiated by identifying each of the participants prior to session's start. During the session, the time and arousal levels are also registered. Finally, it provides a detailed report of overall session outcome, based on previous sessions. As a back-end unit for AR system, all the sampling data are stored in SQLite database in separate tables and in a flat file. These tables are part of an organized structure which represents physical files for fast data access and performance. A backup copy of such data is also copied to comma separated value (CSV) files. Since there can be sensitive data in future application of this technology, in order to protect them in terms of security threat, some table fields data may be encrypted. The data are collected continuously during the therapeutic session by the Bitalino device sensors.

\subsection{Application Architecture}

The application architecture in the Fig. 9, defines the various components and their interactions in the context of AR system. It represents the layered context in between the four main layers: (1) Presentation. (2) Business logic. (3) Data layer. (4) Resource layer. This type of modular organization, highly simplify the software architecture by removing all of the complexity aspects, such as (i.e., excessive coding, deployment, need of component reuse, and scalability). The AR system architecture can be then represented at conceptual level by its functionality in the following diagram below, where the layers are illustrated:

\section{Figure 9: Application Architecture Overview.}

\subsection{Application Implementation}

The build process and the deployment of the Anxiety-AR application, follows a modular structure as in the Fig. 10. The modules point to corresponding package structure, such as: (1) AR framework. (2) Bitalino device handling. (3) Unity code to manage the whole application. (4) Additional libraries. The compilation of the solution was done using Unity3D 2018.2.0f2, which produces either an executable binary file (exe) for windows 8 using .Net framework 3.5, or a single package (apk) for Android supporting platforms 20 (build tools-4.4W). The AR experience was conducted using the ASUS laptop with 15 inch display monitor and G-Force graphic adapter.

The Schematic layout of Anxiety-AR deployment structure is shown below: 
Noname manuscript No.

(will be inserted by the editor)

Figure 10: Anxiety-AR Package Structure for Deployment.

\section{Results}

Taking in consideration some reasonable usage during short period of time, the application has shown to be an additional asset as a non-intrusive tool for the therapists to use in the anxiety disorders interventions. The Anxiety-AR application has a very user-friendly interface, which may comprise a feasible solution for therapists to use in phobia treatment, due to advantages related to the manipulation and management of the adaptive operations in a Bio-cybernetic Loop as shown in the Fig. 11, as well as costly efficient based on its hardware design.

Figure 11: A Cybernetic Loop.

\section{Discussion And Future Work}

In this work, we have built an AR system for AR exposure in the context of snake phobia. The AR system is based on the marker detection algorithm, which detects and tracks an object in the scene. Such a system will assist therapists and the participants, by providing a more intuitive visualization on the location and size of the fear cues, based on electrical signals triggered by ECG and EDA. The system successfully achieved real-time performance on the computer as well as on the Android running device. In the future, we will further evaluate this system with a larger sample and using other objects as fear cues. Furthermore, considering that there is limited information we can obtain from current sensory device hardware, we are planning to build our new sensory system based on a more powerful low cost device hardware with parallel processing capabilities. Beside the potential growth of the current solution to other areas, we also expect to explore other solutions such as AR Oculus, HoloLens, OpenCV and Gesture integration, allowing our system to provide a more powerful visualization and integration capabilities with other technologies in order to scale this technology. Further enhancements must also be made in order to improve the application graphics and reporting interface to adapt to the other contexts, as well as addressing security issues, but also to extend to other mobile applications and platforms, and extend data storage to the cloud.

\section{Conclusions}

The exposure therapy for anxiety disorders is a time consuming treatment for both theapists and patients suffering from anxiety disorders. During the session, the therapist performs observations on each of the participants behavior, reactions, and evaluates the responses to self-report measures based on questionnaire methods. This procedure places great effort in these professionals and can be assisted by a more accurate monitoring of the participants reactions as achieved by this system. Therefore, in order to improve this process during recurring activities of the treatment, one possibility is to automate the therapeutic procedure in order to assist the therapist in this evaluation and to reduce effort during this procedure. To achieve such goal, it was necessary to develop an unified interface application, which could bring together both therapists and participants into a common environment unlike most VR solutions for exposure therapy.

The AR System provides a visually attractive and interactive solution to the therapists since it will deal with all inputs and adapt its behavior based on the values read from sensor data extracted from each participant. All the changes in the participants electrophysiology profiles are reflected directly into a graphic screen, accessible by both therapists and participants. In this case, if each of the participant electrical activity level increase, then the fear cue is zoomed in requiring an effort of the participant to lower the activation levels. For that, the participant must stay in a relaxed state of mind, and the fear cue gets zoom out. After certain time, the application stops data collector, and the report is produced showing the overall session status and progress.

The use of this solution may bring human error rate to a very minimal and the success rate of all the session tasks to a desirable state.

The results of AR system were analyzed by considering both Snake Questionnaire (SNAQ) at the beginning and sensory data that was collected over period of time with each of the participants.

We have identified some Assumptions and Constraints in order to perform initial session. One of such is the access to AR solution, where each of the participant must use graphic user interface (GUI). In order to use the system, he/she needs to be identified on the system prior to starting the session. In the session, the participant needs to be plugged to sensory device in order to collect data. The data must be generated based on the participant electrical activity level variations when subject to a fear cue, either by touch and feel or by showing 


\section{Noname manuscript No.}

(will be inserted by the editor)

animated AR images on the screen. The collected data must be then stored in both, a database and CSV file for later reporting evaluation status. The subsequent sessions must be programmed based on historical data analysis from database. The system must adapt the session parameters according to the electrical activity levels variations, according to the triggers placed in the session. The therapists must be able to follow and interact with the participant during the sessions. The success of exposure is assumed, when the participant shows decreased levels of electrical activity similar to the baseline levels being able to manipulate the fear cue by tactile and visual sensory modalities. Finally, the legal aspects of data security must be followed in order to prevent the security issues. Regarding the limitations, one main limitation was related to the test phase of this system, that was conducted with small set of participants, which might not be sufficient to infer data from each of the participants to further validate the system. Further studies should assess this system with a larger sample of participants, but also with a clinical sample of patients with anxiety disorders to provide consistent data regarding the efficacy of this application as an exposure therapy technique to help the patients overcome anxiety disorders. Overall, this paper provides the preliminary evidence for an adaptive and non-intrusive AR solution for exposure in anxiety disorders.

\section{Acknowledgments}

Preyesse Arquissandás, David Ribeiro Lamas and Jorge Oliveira thanks to COPELABS - Cognitive and PeopleCentric Computing and COFAC / ULHT - Universidade Lusófona de Humanidades e Tecnologias for sponsoring this work.

\section{Abbreviations}

AR: Augmented Reality; ECG: Electrocardiogram; EDA: Electrodermal Activity; GSR: Galvanic Skin Response; HRV: Heart Rate Variability; SNQ: Snake Questionnaire; VR: Virtual Reality

\section{Declarations}

\section{Author's contributions}

Preyesse Arquissandás designed the experiments and participated in the data interpretation. All the other coauthors have edited the manuscript. The author(s) read and approved the final manuscript.

\section{Funding}

This work has also been (partially) funded by FCT strategic project COPELABS UID/04111/2020.

\section{Availability of data and materials}

Datasets generated for this study are available from the corresponding author upon reasonable request.

\section{Code availability (software application or custom code)}

Software application or custom code generated for this study are available from the corresponding author upon reasonable request.

\section{Ethics approval and consent to participate}

The anxiety-AR sessions were approved by all the participants, by a informed consent agreement. 
Noname manuscript No.

(will be inserted by the editor)

\section{Consent for publication}

Not applicable.

\section{Conflicts of interest/Competing interests}

The authors do not have any conflict of interest to report.

\section{Author details}

Preyesse Arquissandás

Copelabs, University Lusófona, Av. Campo Grande 388

1749-024, Lisbon, PORTUGAL

Tel.: +351-21-7515500

E-mail:preyesse.arquissandas@alunos.ulusofona.pt

Url:www.ulusofona.pt

\section{References}

Agrafioti F., Dimitrios H., and Adam A. K., (2012), 'ECG pattern analysis for emotion detection.', IEEE Transactions on Affective Computing, vol. 3, no. 1, pp. 102-115.

American Psychiatric Association, APA (2016), 'Diagnostic and statistical manual of mental disorders.', DSM-V (5th ed., text revision), Washington, DC.

Arquissandás, P., Lamas, D. and Oliveira, J., June (2019), 'Augmented Reality and Sensory Technology for Treatment of Anxiety Disorders.', In 2019 14th Iberian Conference on Information Systems and Technologies (CISTI), pp. 1-4, IEEE.

Baus O., and Bouchard S., (2014), 'Moving from virtual reality exposure-based therapy to augmented reality exposure-based therapy: a review.', Frontiers in Human Neuroscience, vol. 8, article 112.

Bitalino (2020), Available at: http://www.bitalino.pt, Last access on 28-May-2020.

Botella C., Pérez-Ara M.Á., Bretón-López J., Quero S., García-Palacios A., and Baños R.M., (2016), 'In Vivo versus Augmented Reality Exposure in the Treatment of Small Animal Phobia: A Randomized Controlled Trial.', PLoS ONE 11(2): e0148237,doi:10.1371/journal.pone.0148237.

Bradley M., and Lang P., (1994), 'Measuring emotion: The self-assessment manikin and the semantic differential.', J Behav Ther Exp Psychiatry, 2(1), 49-59.

Duenser A., (2014), 'Towards reactive augmented reality exposure treatment.', REHAB.

Emmelkamp P. M. G., Bouman T. K., and Scholing A., (1992), 'Anxiety Disorders.', a Practitioner's Guide.

Emmelkamp P. M., Krijn M., Hulsbosch A. M., de Vries S., Schuemie M. J., and van der Mast C. A., (2002), 'Virtual reality treatment versus exposure in vivo: A comparative evaluation in acrophobia.', Behavior Research and Therapy, 40, 5, pp. 509-516.

Frans S., (2004), 'Hooplot Master Thesis on Agoraphobia', Delft University

Gacek A. and Pedrycz W., (2011), 'ECG signal processing, classification and interpretation: a comprehensive framework of computational intelligence.', Springer Science $\backslash \&$ Business Media. 


\section{Noname manuscript No.}

(will be inserted by the editor)

Gamito P., Oliveira J., Morais D., Rosa P., and Saraiva T., (2011), 'Serious Games for Serious problems: from Ludicus to Therapeuticus.', In J. J. Kim (Ed.) Virtual Reality. InTech, Publishing, pp. 527-548.

Harada Y., Furuya T., Takahashi N., Hasegawa K., Nakazato T., and Ohkura M., (2014), 'Content evaluation of exciting feeling by using biosignals.', Proceeding of AHFE2014, pp. 6931-6936.

Juan M. C., and Botella, (2004), 'An Augmented Reality System for treating psychological disorders: Application to phobia to cockroaches.', Proceedings of the Third IEEE and ACM International Symposium on Mixed and Augmented Reality (ISMAR 2004) 0-7695-2191-6/04 I\$20.00 @ 2004, IEEE.

Juan M. C., and Calatrava J., (2011), 'An augmented reality system for the treatment of phobia to small animals viewed via an optical see-through HMD. Comparison with a similar system viewed via a video see-through HMD.', International Journal of Human-Computer Interaction, vol. 27, no. 5, pp. 436-449.

Juan M. C., and Joele D., (2011), 'A comparative study of the sense of presence and anxiety in an invisible marker versus a marker augmented reality system for the treatment of phobia towards small animals.', International Journal of Human Computer Studies, vol. 69, no. 6, pp. 440-453.

Juan M.C. and Prez D., (2010), 'Using augmented and virtual reality for the development of acrophobic scenarios. Comparison of the levels of presence and anxiety.', Computers and Graphics, vol.34, no. 6, pp. 756766.

Kim K. H., Bang S. W., and Kim S. R., (2004), 'Emotion recognition system using short-term monitoring of physiological signals.', Medical and biological engineering and computing, vol. 42, no.3, pp. 419-427.

Klorman, R., Weerts, T. C., Hastings, J. E., Melamed, B. G., and Lang, P. J. (1974), 'Psychometric descriptions of some specific fear questionnaires.', Behavior Therapy, 5, 401-409.

Muñoz J. E., Gouveia E. R., Cameirão M. S., and Badia S. B. i, (2017), 'PhysioLab a multivariate physiological computing toolbox for ECG, EMG and EDA signals: a case of study of cardiorespiratory fitness assessment in the elderly population.', Multimed. Tools Appl., pp. 1-26.

Muñoz J. E., Rubio E., Cameirao M., and Bermúdez S., (2017), 'The Biocybernetic Loop Engine: an Integrated Tool for Creating Physiologically Adaptive Videogames.', in 4th International Conference in Physiological Computing Systems, Madrid, España.

Nakasone A., Prendinger H., and Ishizuka M., (2005), 'Emotion recognition from electromyography and skin conductance.', Proc. of the 5th International Workshop on Biosignal Interpretation.

NyARToolkit (2020), Available at: http://svn.sourceforge.jp/view/NyARToolkit, Last access on 28-May-2020.

Ohkura M., Goto S., Higo A., and Aoto T., (2011), 'Relation between Kawaii Feeling and Biological Signals.', Transactions of Japan Society of Kansei Engineering, vol. 10, no. 2, pp. 109-114.

Parsons, and Rizzo, (2008), 'Affective Outcomes of Virtual Reality Exposure Therapy for Anxiety and Specific Phobias: A Meta-Analysis.', Journal of Behavior Therapy \\& Experimental Psychiatry, 39, 3, pp. 250-261.

Powers M., and Emmelkamp P., (2008), 'Virtual reality exposure therapy for anxiety disorders: A metaanalysis.', Journal of Anxiety Disorders, 22, 3, pp. 561-569.

Rattanyu K., and Mizukawa M., (2011), 'Emotion Recognition Based on ECG Signals for Service Robots in the Intelligent Space During Daily Life.', Journal Of Advanced Computational Intelligence And Intelligent Informatics, vol. 15, no. 5, pp. 582-591. 


\section{Noname manuscript No.}

(will be inserted by the editor)

Riva G., Molinari E., and Vincelli F., (2002), 'Interaction and presence in the clinical relationship: virtual reality (VR) as communicative medium between patient and therapist.', IEEE Transactions on Information Technology in Biomedicine, 6, 3, pp. 198-205.

Suso-Ribera, C., Fernández-Álvarez, J., García-Palacios, A., Hoffman, H.G., Bretón-López, J., Banos, R.M., Quero, S. and Botella, C., (2019), 'Virtual reality, augmented reality, and in vivo exposure therapy: a preliminary comparison of treatment efficacy in small animal phobia.', Cyberpsychology, Behavior, and Social Networking, 22(1), pp.31-38.

Unity 3D (2020), Available at: http://www.unity.org, Last access on 28-May-2020.

Van Rooij, M., Lobel, A., Harris, O., Smit, N. and Granic, I., May (2016), 'DEEP: A biofeedback virtual reality game for children at-risk for anxiety.', In Proceedings of the 2016 CHI Conference Extended Abstracts on Human Factors in Computing Systems, pp. 1989-1997.

Wiederhold M. D., (2012), 'Cyber Therapy - Augmented Reality in Healthcare.', The Virtual Reality Medical Center - www.vrphobia.com.

Wolitzky-Taylor K. B., Horowitz D. J., Powers M. B., and Telch M. J., (2008), 'Psychological approaches in the treatment of specific phobias: A meta-analysis.', Clinical Psychology Review, 28(6), 1021-1037.

Wrzesien M., Burkhardt J. M., Alcañiz M., and Botella C., (2011), 'How technology influences the therapeutic process: a comparative Field evaluation of augmented reality and in vivo exposure therapy for phobia of small animals.', in Proceedings of the Human-Computer Interaction (INTERACT 11-Lisbon-Portugal), pp. 523-540.

\section{Table of Contents}

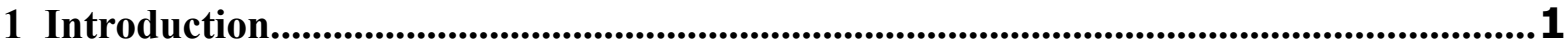

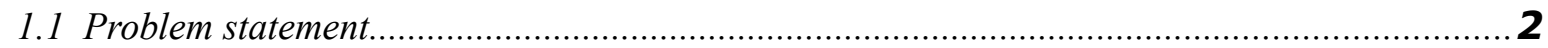

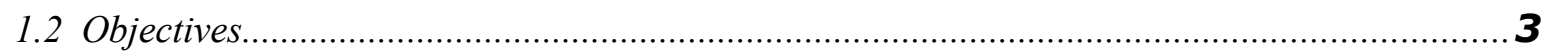

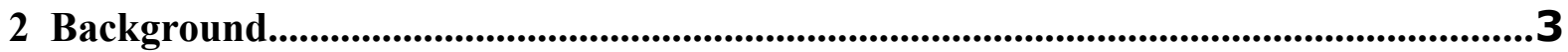

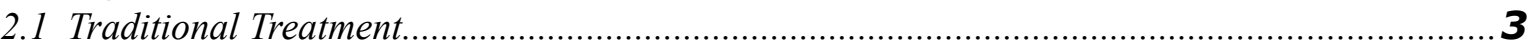

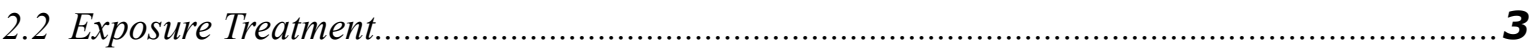

2.3 AR System for Treating Anxiety Disorders through psychophysiological assessments...............3

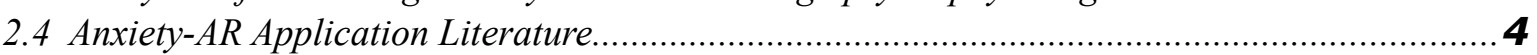

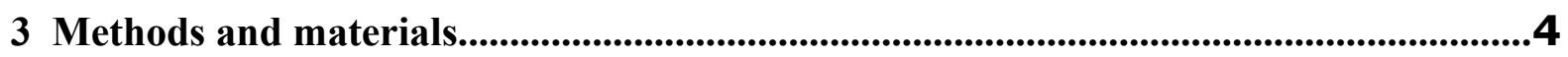

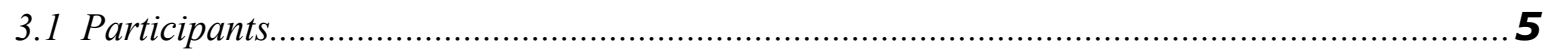

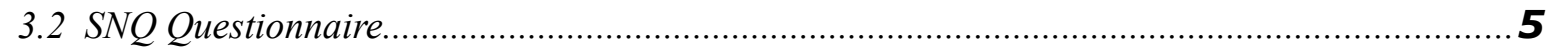

3.3 AR Exposure

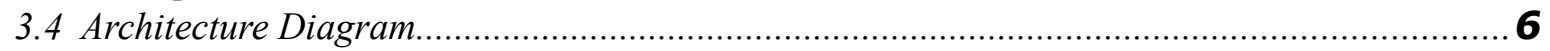

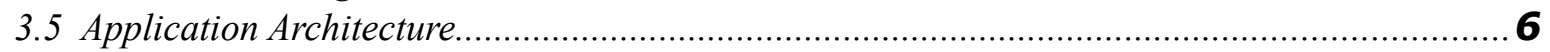

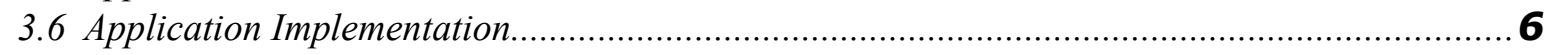

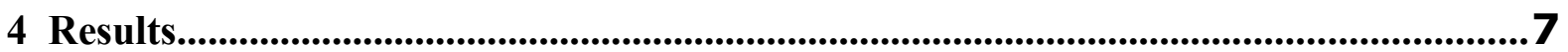

5 Discussion And Future Work........................................................................................

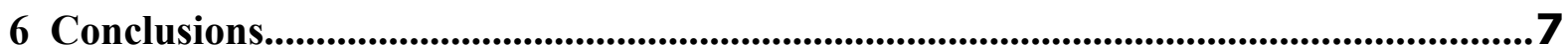




\section{Illustration Index}

Figure 1: Summary responses of SNQ by Origin/Gender.......................................................13

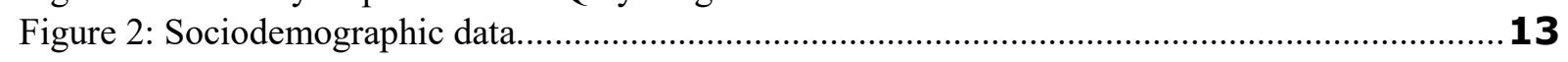

Figure 3: Psychophysiological data sample from assessments moments.......................................13

Figure 4: Psychophysiological data analysis from four (4) data sessions of 5 minutes each one........14

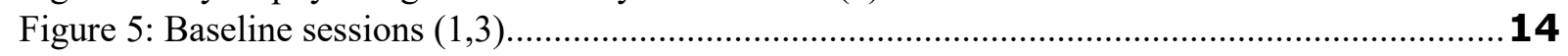

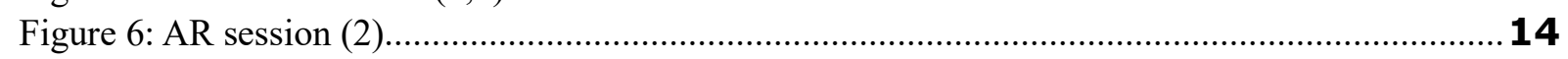

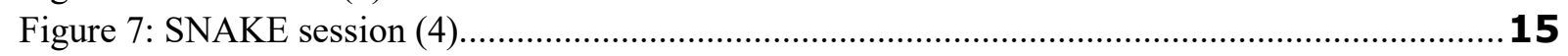

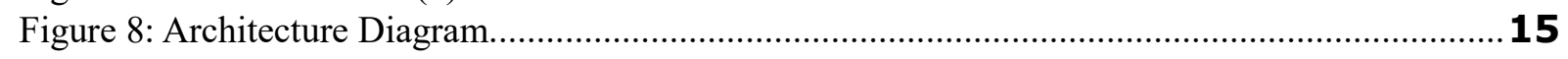

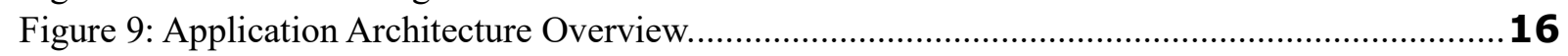

Figure 10: Anxiety-AR Package Structure for Deployment..........................................................16

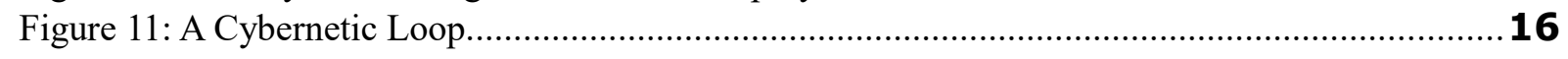

\section{Index of Tables}

Table 1: Snake Questionnaire (SNAQ) Sample - (Klorman et al., 1974).......................................17

Table 2: The Questionnaire overall sociodemographic coverage................................................17

Table 3: The Snake Questionnaire (SNQ) results of the participants.....................................................17 
Noname manuscript No.

(will be inserted by the editor)

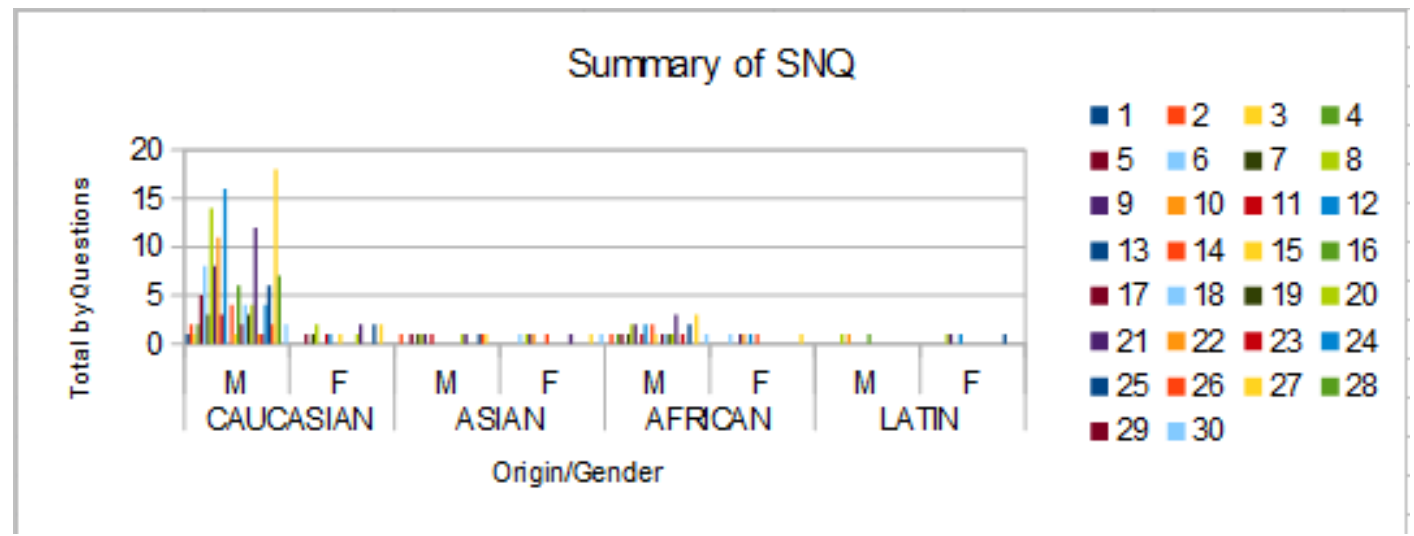

Figure 1: Summary responses of SNQ by Origin/Gender.

TOTAL EY QLESTIONS

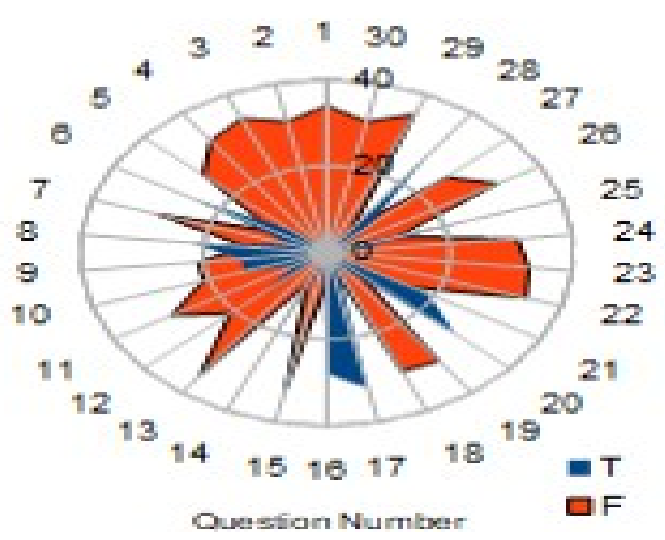

Figure 2: Sociodemographic data.

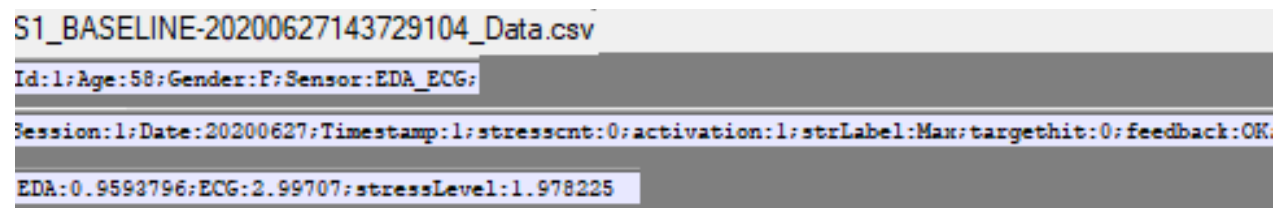

Figure 3: Psychophysiological data sample from assessments moments. 
Noname manuscript No.

(will be inserted by the editor)

Session Analysis

Target $\mathrm{Hit}$

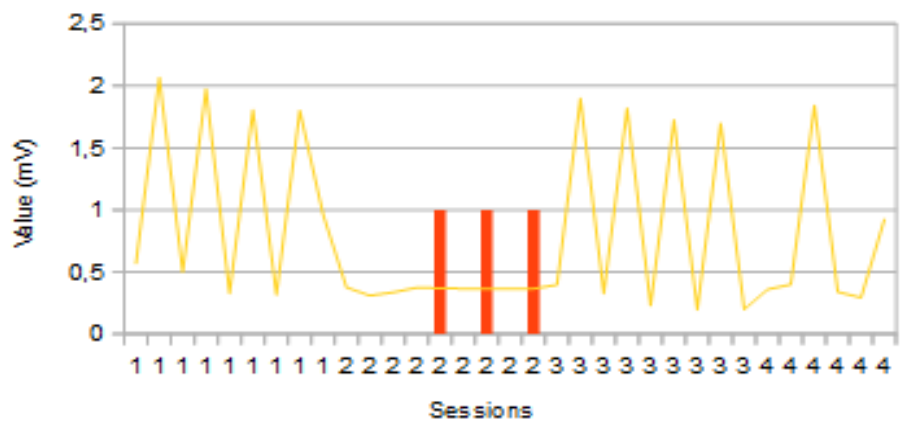

Target hit stresslevel

Figure 4: Psychophysiological data analysis from four (4) data sessions of 5 minutes each one.

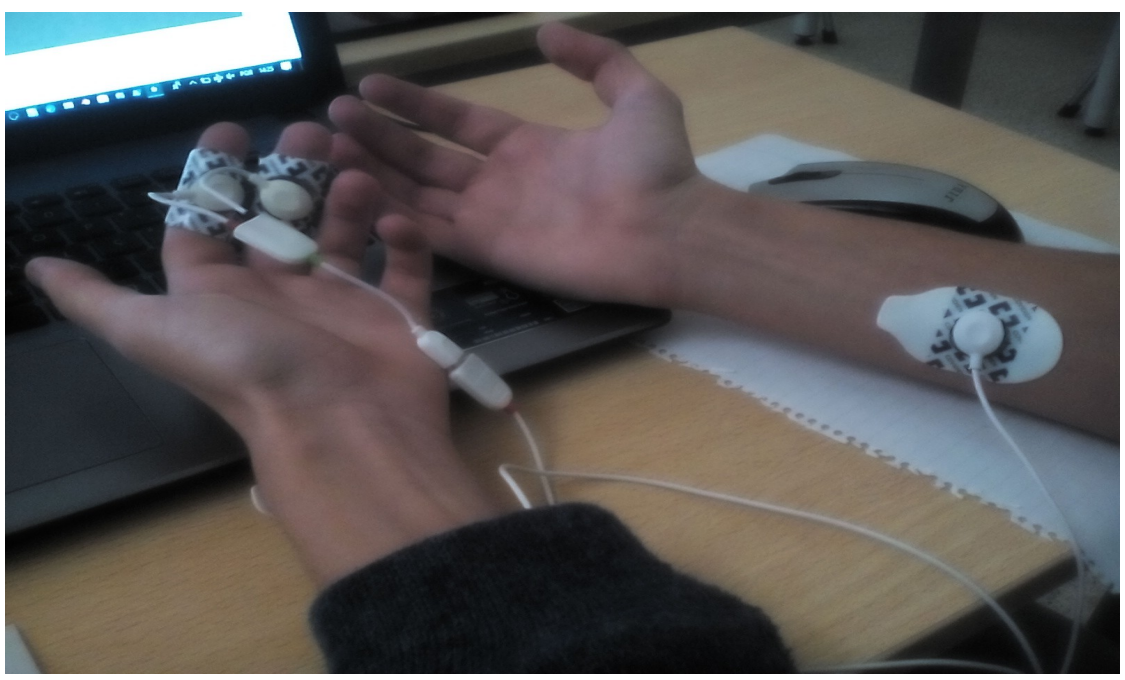

Figure 5: Baseline sessions $(1,3)$.

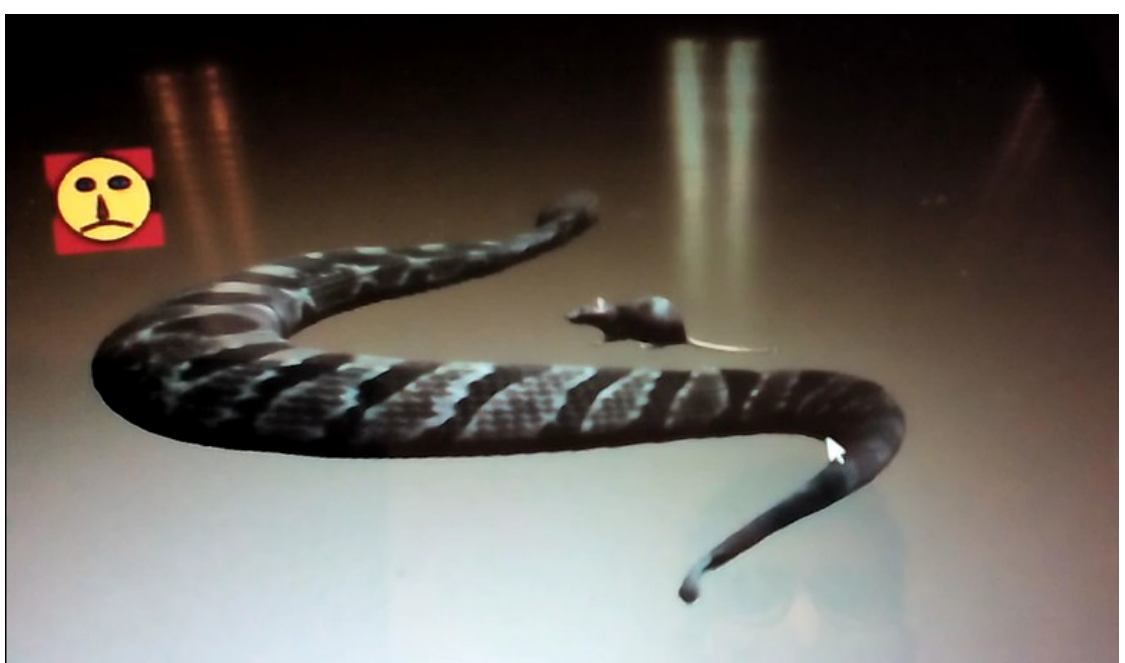

Figure 6: AR session (2). 
Noname manuscript No.

(will be inserted by the editor)

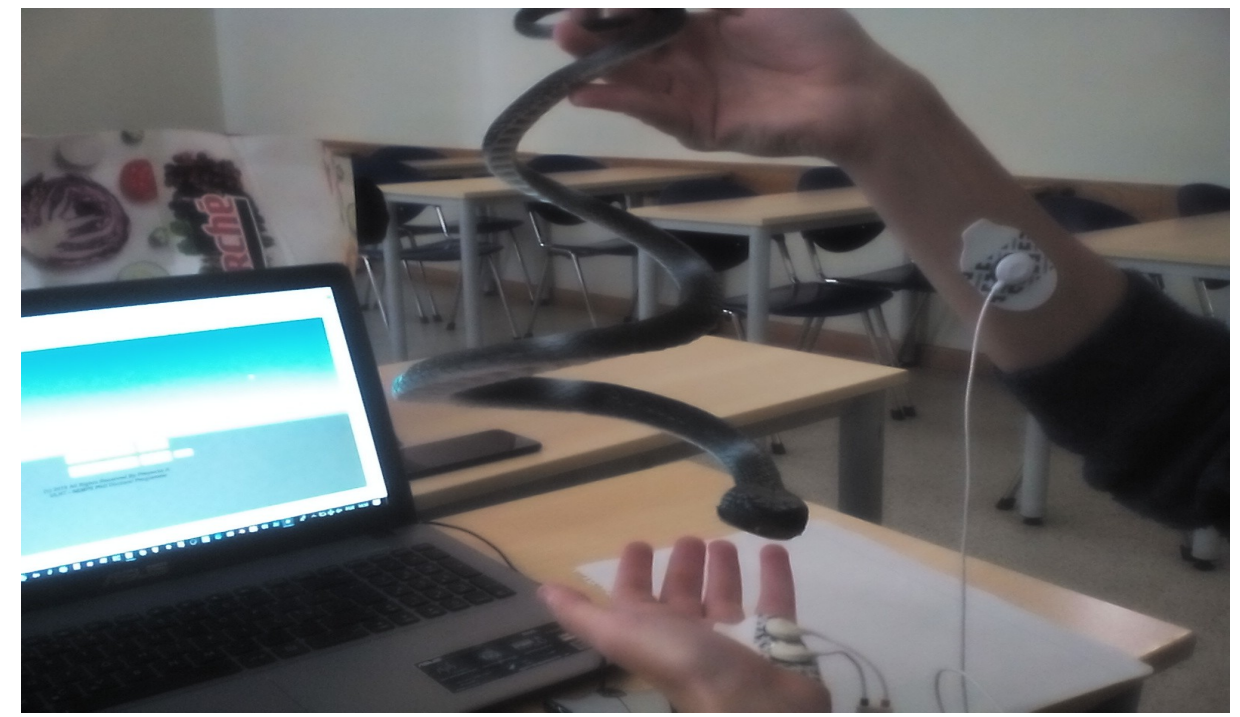

Figure 7: SNAKE session (4).

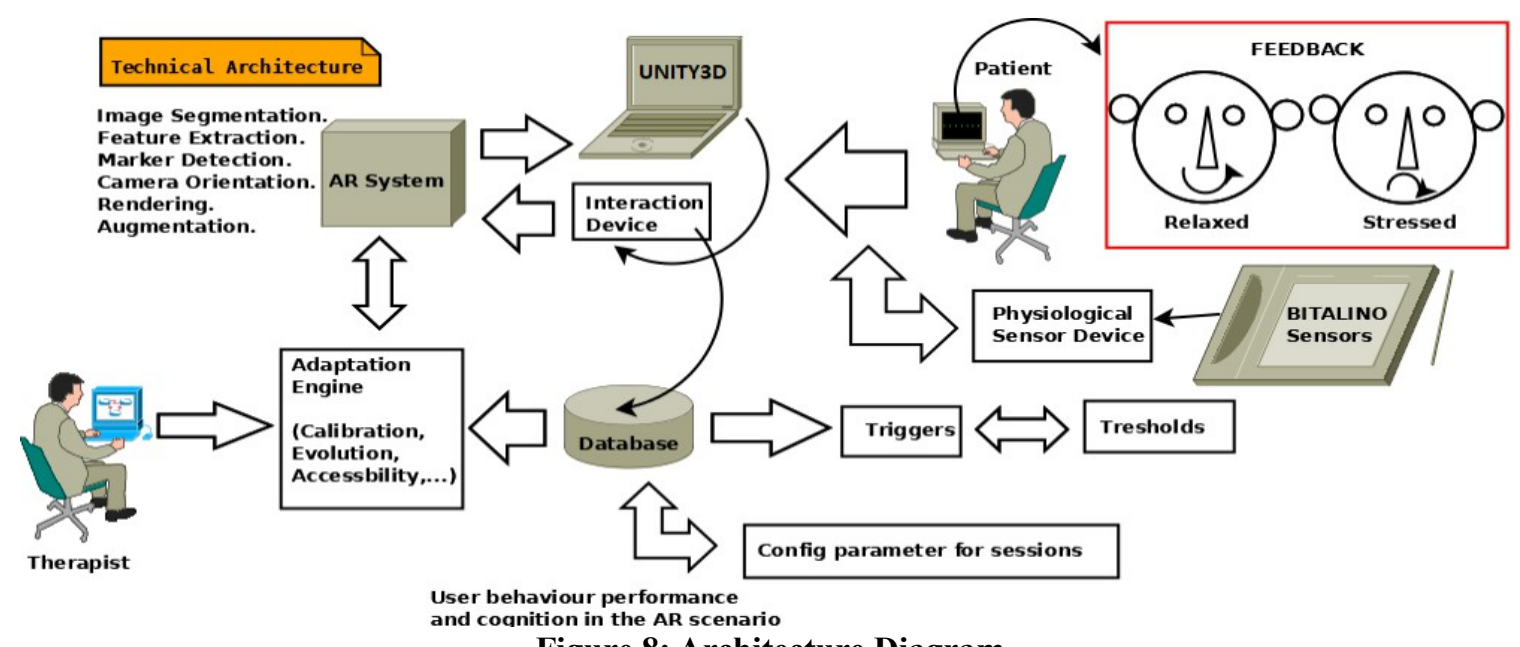

Figure 8: Architecture Diagram. 
Noname manuscript No.

(will be inserted by the editor)

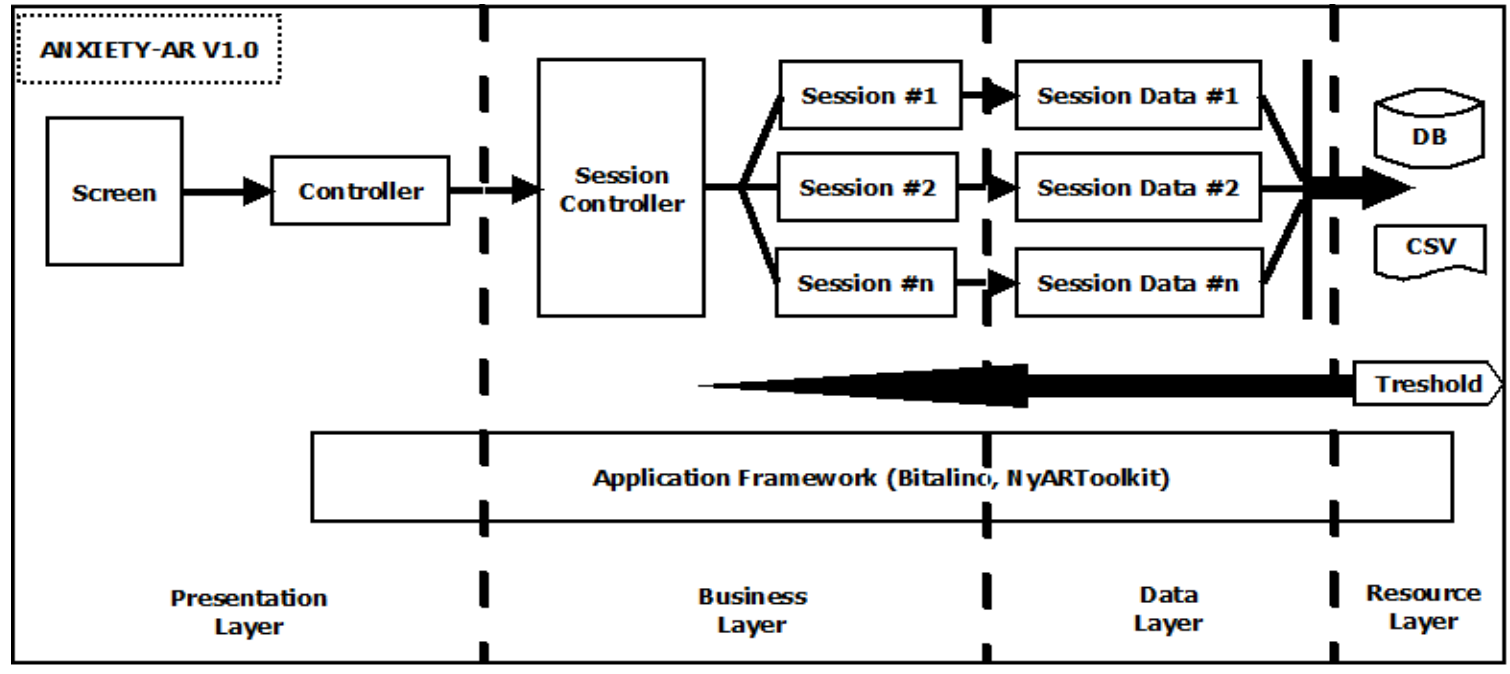

Figure 9: Application Architecture Overview.

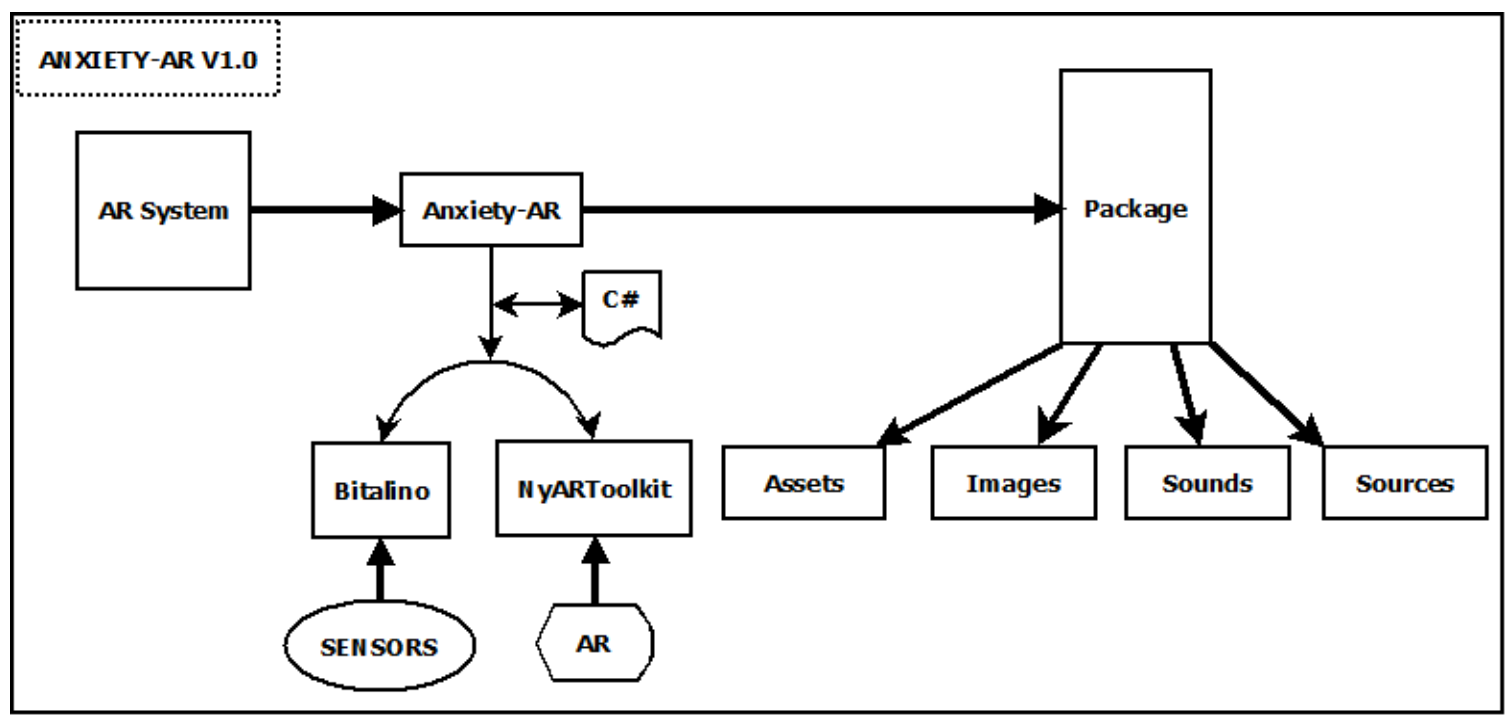

Figure 10: Anxiety-AR Package Structure for Deployment.

A Cybernetic Loop

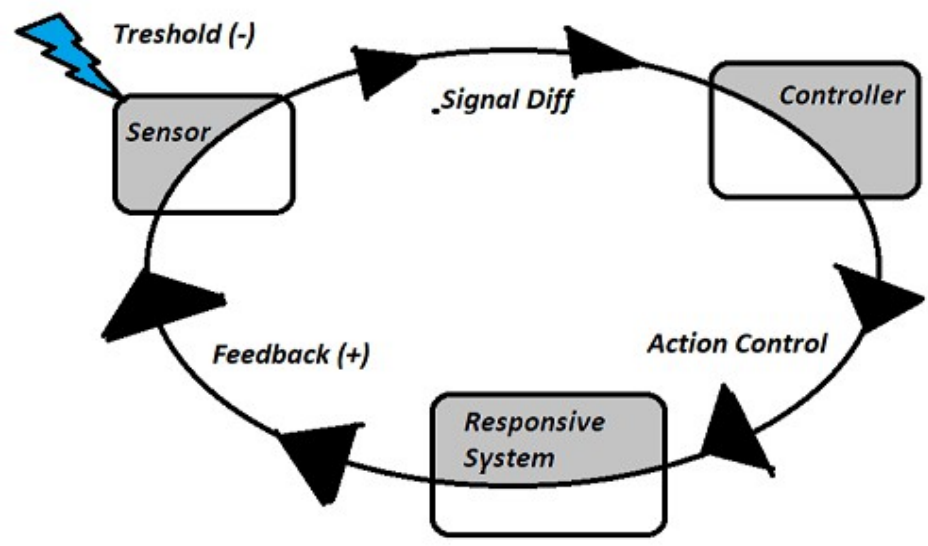

Figure 11: A Cybernetic Loop. 


\section{Noname manuscript No.}

(will be inserted by the editor)

Table 1: Snake Questionnaire (SNAQ) Sample - (Klorman et al., 1974).

Statements

1. I avoid going to parks or on camping trips because there may be snakes about.

2. I would feel some anxiety holding a toy snake in my hand.

3. If a picture of a snake appears on the screen during a motion picture, I turn my head away.

4. I dislike looking at pictures of snakes in a magazine.

5. Although it may not be so, I think of snakes as slimy.

Table 2: The Questionnaire overall sociodemographic coverage.

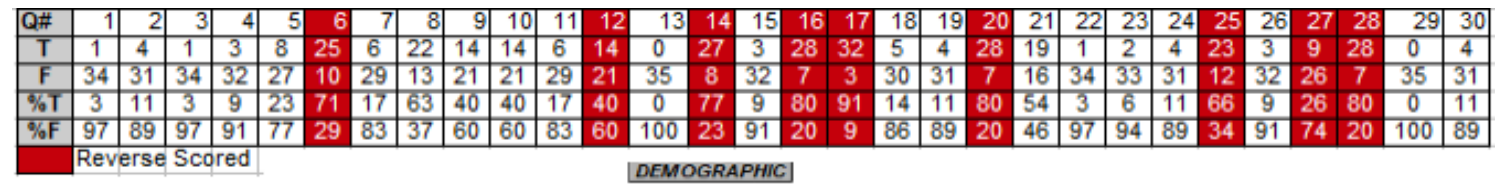

\begin{tabular}{|l|r|}
\hline AGE & \# \\
\hline 19 & 3 \\
\hline 20 & 18 \\
\hline 21 & 6 \\
\hline 22 & 3 \\
\hline 23 & 2 \\
\hline 24 & 2 \\
\hline 29 & 1 \\
\hline
\end{tabular}

\begin{tabular}{|l|r|}
\hline ORIGIN & \# \\
\hline CAUCASIAN & 26 \\
\hline ASIAN & 2 \\
\hline AFRICAN & 5 \\
\hline LATIN & 2 \\
\hline
\end{tabular}
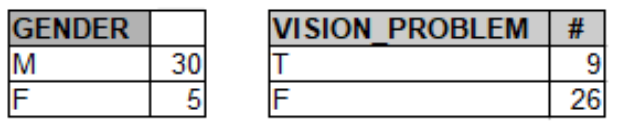

Table 3: The Snake Questionnaire (SNQ) results of the participants.

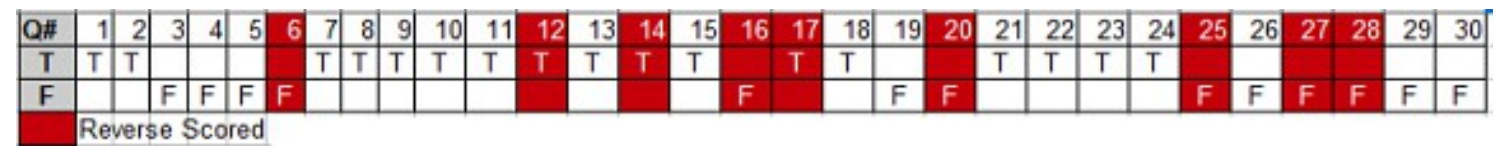

